\title{
Circadian-temporal context and latent inhibition of conditioned taste aversion: Effect of restriction in the intake of the conditioned taste stimulus
}

\author{
Andrés Molero-Chamizo ${ }^{1,2}$
}

Published online: 11 November 2016

(C) Psychonomic Society, Inc. 2016

\begin{abstract}
Latent inhibition of conditioned taste aversion (CTA) is sensitive to changes in the temporal context. A change in the time of day of conditioning with respect to the time of day of the preexposure can disrupt the latent inhibition. This contextual change in the time of day may reveal a temporal specificity of latent inhibition. The optimum procedure to induce this temporal specificity is not well established. For example, it has been shown that a long period of habituation to temporal contexts is one factor that can determine the effect. However, the experimental conditions on the conditioning day that facilitate this phenomenon are unknown. The aim of this study is to elucidate whether a restriction in the intake of the conditioned taste stimulus affects the temporal specificity of latent inhibition. Two main groups of Wistar rats were tested in a latent inhibition of CTA paradigm, in which the temporal specificity of this phenomenon was analyzed by a change in the time of day of conditioning. The intake of the taste stimulus was restricted in the conditioning day in one of the groups, but this restriction was not applied in the other group. The results indicated temporal specificity of latent inhibition only in the group without restriction, but not in the group with limitation in the intake of the taste stimulus during conditioning. These findings can help to elucidate the characteristics of the procedure to induce temporal specificity of latent inhibition.
\end{abstract}

Andrés Molero-Chamizo

andres.molero@dpsi.uhu.es

1 Present address: Department of Psychobiology, University of Granada, Campus Cartuja, Granada 18071, Spain

2 Department of Psychology, Psychobiology Area, University of Huelva, Campus El Carmen, 21071 Huelva, Spain
Keywords Conditioned taste aversion · Latent inhibition · Taste stimulus restriction · Temporal context

The magnitude of a conditioned taste aversion (CTA) can be modulated by a change in the time of day between conditioning and test (Manrique, Gámiz, Morón, Ballesteros, \& Gallo, 2009; Morón et al., 2002). This procedure shows that animals tested in temporal contexts different from that of the conditioning acquire less aversion, which is similar to what has been consistently described by changing the spatial context (Boakes, Elliot, Swinbourne, \& Westbrook, 1997; Bonardi, Honey, \& Hall, 1990; Bouton, 1993; Bouton, Westbrook, Corcoran, \& Maren, 2006; González, Garcia-Burgos, \& Hall, 2012; Pearce \& Bouton, 2001; Rosas \& Bouton 1997). Similarly, the latent inhibition of CTA can be disrupted by changes in the time of day between preexposure and conditioning (Manrique et al., 2004; Molero et al., 2005). Nevertheless, the characteristics of the procedure that facilitate this temporal specificity of the latent inhibition (or dependency of latent inhibition on the temporal context - time of dayof preexposure and conditioning) are not entirely known. Long periods of habituation to different temporal contexts seem to facilitate this phenomenon (Molero-Chamizo, 2013). This effect might mean that contextual familiarity and associative strength of the conditioned stimulus are relevant factors for the specificity of latent inhibition, as has been described in contextual learning (Pearce \& Bouton, 2001). Moreover, a restriction in the intake of the conditioned taste stimulus may also influence the conditioning process and therefore may affect the temporal specificity of latent inhibition. As a result, a reduced processing of the taste stimulus during conditioning would prevent the effect of temporal specificity. For example, Morón et al. (2002) applied a restriction in the intake of the taste stimulus during 
conditioning, resulting in a modulation of the taste aversion when tested in a different time of day from that of the conditioning. However, by 4 days of habituation to the temporal context, Molero-Chamizo (2013) found temporal specificity of the latent inhibition of CTA without considering any restriction in the amount of taste stimulus during conditioning. Although in both cases different processes were measured (CTA or latent inhibition of CTA), it seems necessary to clarify whether a restriction in the amount of taste stimulus during conditioning (and therefore an external control of the intake) facilitates or hinders the temporal specificity of the latent inhibition of CTA, similar to that described in other learning processes (De la Casa \& Lubow, 1995; González, Morillas, \& Hall, 2015; Lukoyanov, Pereira, Mesquita, \& Andrade, 2002). Thus, the aim of this study is to analyze the effects of restriction versus no restriction in the intake of the taste stimulus at the stage of conditioning on the temporal specificity of the latent inhibition of CTA.

A similar procedure that has previously shown such temporal specificity (Molero-Chamizo, 2013) was used in this study. However, some of the animals had limited access to the intake of the taste stimulus in the day of conditioning (group with restriction), and the remaining animals had no restriction in the intake of the taste stimulus during conditioning (group without restriction), except the time for recording that was common to all animals. If the temporal specificity of the latent inhibition of CTA depends on the free control of the intake during conditioning, then in animals with restriction in the taste stimulus (external control of the intake) the latent inhibition will not be specific of the temporal context; that is, these animals will show latent inhibition regardless of a change in the time of day between preexposure and conditioning.

\section{Method}

\section{Subjects}

Fifty adult male Wistar rats, weighing between 280 and 300 g, were individually housed in boxes measuring $30 \mathrm{~cm} \times 15 \mathrm{~cm}$ $\times 30 \mathrm{~cm}$. All of the animals were exposed to a daily 12 hours light/dark cycle (lights on from 9:00 to 21:00), and the temperature conditions were kept constant at $23{ }^{\circ} \mathrm{C}$. Throughout the procedure, food was provided ad libitum, and the availability of fluid was restricted to two daily $15 \mathrm{~min}$ sessions, one in the morning (10:00) and one in the evening (20:00), for all animals. Twenty-four of them were exposed to a limited amount of taste stimulus ( $5 \mathrm{ml}$ ) for $15 \mathrm{~min}$ during conditioning (group with restriction), and 26 rats had free access to the intake of the taste stimulus for $15 \mathrm{~min}$ during conditioning (group without restriction). The procedure was approved by the Ethics Committee for Animal Research of the University of Granada and was conducted in accordance with both the NIH Publications (No. 80-23) of the National Institutes of Health Guide (United States) for the Care and Use of Laboratory Animals (1996 revision) and the European Community Council Directive of November 24, 1986 (86/ 609/EEC). The National Legislation, in agreement with this Directive, is defined in Royal Decree No. 1201/2005.

\section{Procedure}

In the group with restriction in the intake of the taste stimulus, rats were in turn distributed among the following four subgroups: PE-S (preexposed to the taste, PE, and same time of day, S, for conditioning and testing, $n=8$ ), PE-D (preexposed to the taste, $\mathrm{PE}$, and different time of day, $\mathrm{D}$, for conditioning and testing, $n=8$ ), NPE-S (nonpreexposed to the taste, NPE, and same time of day, $\mathrm{S}$, for conditioning and testing, $n=4$ ), NPE-D (nonpreexposed to the taste, NPE, and different time of day, $\mathrm{D}$, for conditioning and testing, $n=4)$ ). In the group without restriction, animals were distributed among the same four subgroups: PE-S $(n=8)$, PE-D $(n=8)$, NPE-S $(n=5)$, NPE-D $(n=5)$.

All animals were water restricted and received two 15-min sessions of access to water per day, as described above, over 4 days to facilitate the differentiation of the temporal contexts (morning vs. evening). After this period, the procedure had three main stages (preexposure, conditioning, and testing). With respect to the preexposure stage, all rats had access to water in the morning session (15 $\mathrm{min}$ ) for 2 days. The nonpreexposed subgroups (NPE-S and NPE-D) also received water in the evening session (15 $\mathrm{min}$ ) of these 2 days, whereas the preexposed subgroups (PE-S and PE-D) were exposed to a sodium chloride solution dissolved in water (saline 1\%) for 15 $\min$. On the day following the last preexposure, conditioning occurred in the morning session for the PE-D and NPE-D subgroups. These subgroups of the group without restriction in the intake of the taste stimulus were exposed to saline for $15 \mathrm{~min}$ in the morning, and the amounts ingested were recorded. The PE-D and NPE-D subgroups of the group with restriction were exposed to $5 \mathrm{ml}$ of saline for $15 \mathrm{~min}$ in the morning, and the amounts ingested were recorded. This was the only difference in the procedure between the groups with and without restriction in the intake of the taste stimulus. Twenty minutes later, all animals received an injection of lithium chloride ( $\mathrm{LiCl} 0.15 \mathrm{M}, 2 \%$ of body weight, intraperitoneally). Water was available for $15 \mathrm{~min}$ in the evening session. In contrast, the PE-S and NPE-S subgroups received conditioning in the evening session (with saline restriction to $5 \mathrm{ml}$ only in the group with restriction) and water (15 $\mathrm{min})$ in the morning session. After 1 day of recovery with water $(15 \mathrm{~min})$ in the morning and evening sessions, the response to saline (testing stage) was tested in all animals in the evening session for 5 
Table 1 Behavioral procedure (preexposure, conditioning, and testing stages)

\begin{tabular}{|c|c|c|c|}
\hline GROUP & PE1-2/Wpm & CTA & $\mathrm{T} 1-\mathrm{T} 5 \mathrm{pm}$ \\
\hline PE-D & $\begin{array}{l}\text { Water am } \\
\text { Saline } 1 \% \text { pm }\end{array}$ & Saline $+\mathrm{LiCl}$ ip am & Saline pm \\
\hline PE-S & $\begin{array}{l}\text { Water am } \\
\text { Saline } 1 \% \text { pm }\end{array}$ & Saline $+\mathrm{LiCl}$ ip pm & Saline pm \\
\hline NPE-D & Water am-pm & Saline $+\mathrm{LiCl}$ ip am & Saline pm \\
\hline NPE-S & Water am-pm & Saline $+\mathrm{LiCl}$ ip pm & Saline $\mathrm{pm}$ \\
\hline
\end{tabular}

Note. $\mathrm{PE} 1-2 / \mathrm{Wpm}=$ Days 1 and 2 of water or preexposure to saline in the evening session; $\mathrm{CTA}=$ conditioning day; $\mathrm{T} 1-\mathrm{T} 5 \mathrm{pm}=$ tests days in the evening session; PE-D = preexposed subgroup in the "different (D)" context (CTA in the morning session); PE-S = preexposed subgroup in the "same (S)" context (CTA in the evening session); NPE-D = nonpreexposed subgroup in the "different" context; NPE-S = nonpreexposed subgroup in the "same" context. For brevity, the morning consumption after CTA is not represented. Animals with saline restriction in the conditioning day (group with restriction) were exposed to $5 \mathrm{ml}$ of saline on the conditioning day, and the remaining of the procedure was identical to that of animals without saline restriction on the conditioning day (group without restriction).

days. Water was available for $15 \mathrm{~min}$ in the morning sessions. Table 1 summarizes the behavioral procedure.

\section{Statistical analysis}

The saline consumption on the conditioning day was analyzed using a $2 \times 2 \times 2$ factorial design with three between-subjects factors, the first being preexposure (preexposure vs. nonpreexposure), the second factor being context (same "S" vs. different " $D$ " temporal context of conditioning), and the third factor being restriction in the intake of the taste stimulus (restriction vs. no restriction). The saline consumption across the test days was analyzed by a $2 \times 2 \times 2 \times 5$ repeatedmeasures ANOVA. Bayesian model comparison was conducted because of the sample size of some subgroups. When the interactions were significant, Newman-Keuls post hoc tests were applied to analyze differences. The critical level of significance in all tests was set to $p<.05$. The analyses were carried out using SPSS software.

\section{Results}

The results indicate that a change of the time of day between preexposure and conditioning (PE-D subgroup) disrupts the latent inhibition of CTA in animals without restriction in the intake of the taste stimulus. In contrast, latent inhibition was acquired when preexposure and conditioning were performed at the same time of day (PE-S subgroup) in these animals. Figure 1 represents the mean consumption by subgroups on the preexposure, conditioning, and test days for animals without restriction.

Regarding the group with restriction in the intake of the taste stimulus during conditioning, unlike the group without restriction, the results indicate that a change of the time of day between preexposure and conditioning (PE-D subgroup) does not disrupt the latent inhibition of CTA. Latent inhibition was acquired by all subgroups regardless of a change in the temporal context. For the group with restriction, Fig. 2 represents the mean consumption by subgroups on the pre-exposure, conditioning and test days.

An ANOVA of the mean consumption on the conditioning day indicated no significant effect of the interaction between preexposure, context, and restriction, $F(1,42)=2.77, p=.1$. The repeated-measures ANOVA conducted to evaluate the

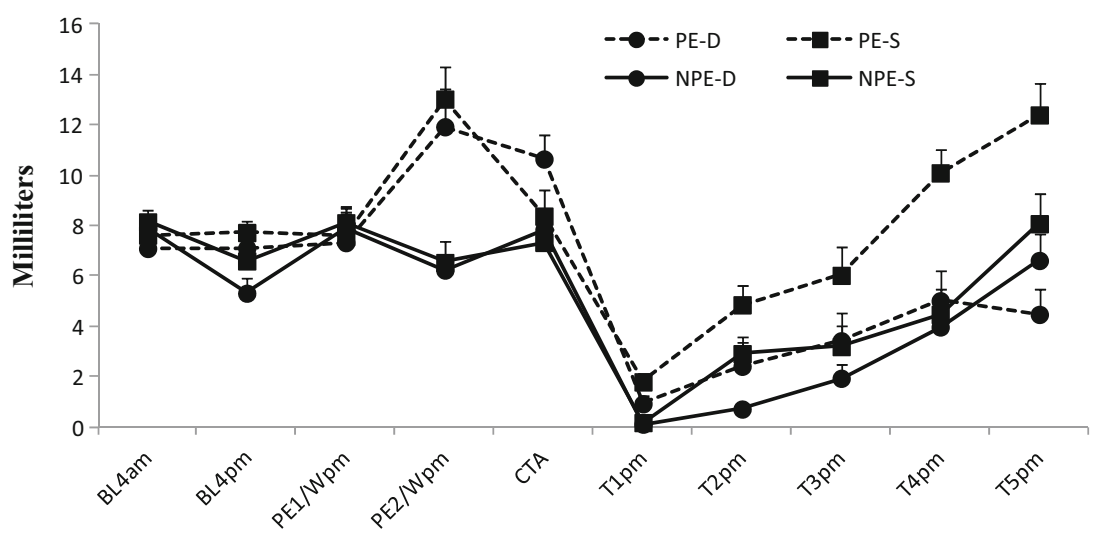

Fig. 1 Mean consumption of saline/water (in $\mathrm{ml}$ ) by subgroups without restriction in the intake of the taste stimulus at different stages of the behavioral procedure, and standard deviation. BL4 = last of the 4 days of baseline; am = morning session; $\mathrm{pm}$ = evening session; $\mathrm{PE} 1-2 / \mathrm{Wpm}=$ Days 1 and 2 of water or preexposure to saline in the evening session; $\mathrm{CTA}=$ conditioning day; $\mathrm{T} 1-\mathrm{T} 5 \mathrm{pm}=$ tests days in the evening session; $\mathrm{PE}-\mathrm{D}=$ preexposed subgroup in the "different (D)" context (CTA in the morning session); $\mathrm{PE}-\mathrm{S}$ = preexposed subgroup in the "same (S)" context (CTA in the evening session); NPE-D = nonpreexposed subgroup in the "different (D)" context; NPE-S = nonpreexposed subgroup in the "same (S)" context. For brevity, the morning consumption after CTA is not represented. The analysis of the main interaction shows that the PE-S, but not the PE-D, subgroup without restriction acquired latent inhibition. 


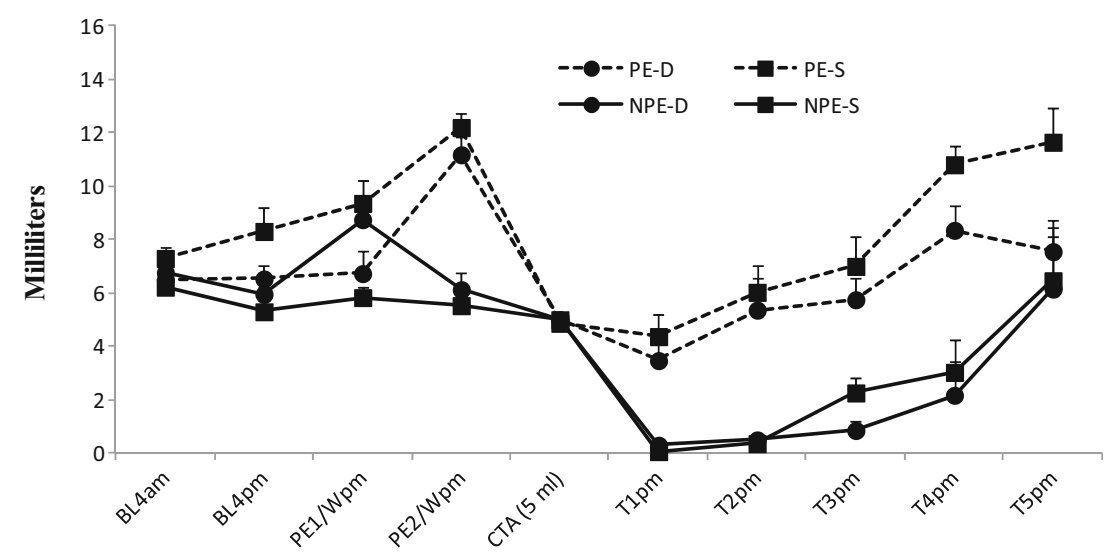

Fig. 2 Mean consumption of saline/water (in $\mathrm{ml}$ ) by subgroups with restriction in the intake of the taste stimulus at different stages of the behavioral procedure, and standard deviation. BL4 = last of the 4 days of baseline; $\mathrm{am}=$ morning session; $\mathrm{pm}=$ evening session; $\mathrm{PE} 1-2 / \mathrm{Wpm}=$ Days 1 and 2 of water or preexposure to saline in the evening session; $\mathrm{CTA}=$ conditioning day (saline restriction to $5 \mathrm{ml}$ ); T1-T5pm = tests days in the evening session; $\mathrm{PE}-\mathrm{D}=$ preexposed subgroup in the "different (D)" context (CTA in the morning session); PE-S = preexposed subgroup in the "same (S)" context (CTA in the evening session); NPE-D = nonpreexposed subgroup in the "different (D)" condition; NPE-S = nonpreexposed subgroup in the "same (S)" condition. The analysis of the main interaction indicates that all preexposed subgroups (PE-D and PE-S) with restriction acquired latent inhibition. mean consumption of each group throughout the 5 test days revealed a significant effect of the interaction between preexposure, context, restriction, and test $F(4,39)=21.72, p$ $=.001$. Newman-Keuls post hoc tests indicated no significant effect of the preexposure factor in the different context for animals without restriction $(p>.05)$, indicating that the PED subgroup did not show latent inhibition. There was a significant effect of the preexposure factor in the same context ( $p$ $<.05$ ) for these animals, which indicates that the PE-S subgroup without restriction acquired latent inhibition. NewmanKeuls post hoc tests also showed a significant effect of the context factor in the preexposed animals without restriction, indicating that the mean consumption of the PE-S subgroup was higher than that of the PE-D subgroup $(p<.05)$. In contrast, there was a significant effect of the preexposure factor for animals with restriction $(p<.05)$, which indicates that all preexposed subgroups (PE-D and PE-S) with restriction acquired latent inhibition. Data from Bayesian model comparison conducted to evaluate statistical power of the results are shown in Table 2. Bayesian model comparison was implemented for the repeated-measures ANOVA, including test days as repeated measures (5 days) and group as betweensubjects factor (with comparisons for the resulting groups from the Preexposure $\times$ Context $\times$ Restriction interaction). These comparisons support the findings of the repeatedmeasures ANOVA, in showing that a model including a Test Days $\times$ Group interaction provides a better fit to the data than a model with main effects but no interaction: the Bayes Factor in favor of the interaction model is given by $2.710 \mathrm{e} 199 /$ $2.743 \mathrm{e} 100=9.88 \mathrm{e} 98$.

Table 2 Bayesian model comparison for the repeated-measures ANOVA

\begin{tabular}{|c|c|c|c|c|c|}
\hline $\begin{array}{l}\text { Models } \\
\text { Null model (incl. subject) }\end{array}$ & $\begin{array}{l}P(\mathrm{M}) \\
0.250\end{array}$ & $\begin{array}{l}\mathrm{P}(\mathrm{M} \mid \text { data }) \\
4.092 \mathrm{e}-74\end{array}$ & $\begin{array}{l}\mathrm{BF}_{\mathrm{M}} \\
1.228 \mathrm{e}-73\end{array}$ & $\begin{array}{l}\mathrm{BF}_{10} \\
1.000\end{array}$ & $\%$ error \\
\hline Test & 0.250 & 0.427 & 2.236 & $1.044 \mathrm{e}+73$ & 0.438 \\
\hline CTA & 0.250 & $3.669 \mathrm{e}-74$ & $1.101 \mathrm{e}-73$ & 0.897 & 0.952 \\
\hline Test + CTA & 0.250 & 0.573 & 4.025 & $1.400 \mathrm{e}+73$ & 3.498 \\
\hline Null model (incl. subject) & 0.200 & $3.690 \mathrm{e}-200$ & $1.476 \mathrm{e}-199$ & 1.000 & \\
\hline Test & 0.200 & $3.879 \mathrm{e}-127$ & $1.551 \mathrm{e}-126$ & $1.051 \mathrm{e}+73$ & 0.630 \\
\hline Group & 0.200 & $1.316 \mathrm{e}-188$ & $5.265 \mathrm{e}-188$ & $3.567 \mathrm{e}+11$ & 0.495 \\
\hline Test + Group & 0.200 & $1.012 \mathrm{e}-99$ & $4.048 \mathrm{e}-99$ & $2.743 e+100$ & 0.565 \\
\hline Test + Group + Test $\times$ Group & 0.200 & 1.000 & $3.952 \mathrm{e}+99$ & $2.710 e+199$ & 0.889 \\
\hline
\end{tabular}

Note . $\mathrm{BF}=$ Bayesian $\mathrm{F} ; \mathrm{CTA}=$ conditioning day; Group = resulting groups from the Preexposure $\times$ Context $\times$ Restriction interaction; $\mathrm{M}=$ comparison model; $P=$ probability for models. All models include subject. Considering null model as 0 , main effects as 1 and interaction effects as $2: \mathrm{BF}_{1,2}=\mathrm{BF}_{1,0} *$ $\mathrm{BF}_{0,2}=\mathrm{BF}_{1,0} / \mathrm{BF}_{2,0}=2.743 \mathrm{e}+100 / 2.710 \mathrm{e}+199=1.012 \mathrm{e}-99$. These comparisons support the findings of the repeated-measures ANOVA, in showing that a model including a Test Days $\times$ Group interaction provides a better fit to the data than a model with main effects but no interaction: the Bayes factor in favor of the interaction model is given by $2.710 \mathrm{e} 199 / 2.743 \mathrm{e} 100=9.88 \mathrm{e} 98$. 


\section{Discussion}

The results of this study indicate that the latent inhibition of CTA may be disrupted by changes in the temporal context between preexposure and conditioning. This temporal specificity of the latent inhibition seems only to occur when animals have free access to the taste stimulus during the exposure interval, but not when the taste stimulus is restricted and limits the intake behavior of animals. Throughout the test days, the consumption of the PE-S subgroup in the group without restriction in the intake of the taste stimulus was higher than that of the PE-D and NPE-S subgroups. However, the consumption of the PE-D subgroup was not different from that of the NPE-D subgroup. Thus, only animals in which the temporal context remained the same between preexposure and conditioning (PE-S subgroup) showed latent inhibition in the group without restriction. Although the sample size in some subgroups was limited, statistical analyses of the data suggest that the temporal context has an effect on latent inhibition when no restriction in the intake is applied during conditioning. It can be argued that, assuming the statistical power of the main interaction shown by the Bayesian model comparison, these results support the possibility that a free control of the intake during conditioning facilitates the temporal specificity of the latent inhibition in the CTA paradigm, and an external control of the intake during conditioning affects the temporal specificity of this phenomenon. Considering these limitations, these findings seem to be consistent with previous studies in which different contextual cues have been used. For example, latent inhibition was reduced in a study in which external cues (and other variables such as the number of stimulus preexposures) were changed between preexposure and testing (De la Casa \& Lubow, 2001), indicating that latent inhibition is sensitive to contextual changes in different stages of the experimental procedure. In the CTA paradigm, latent inhibition attenuation after an external context change between preexposure and conditioning/testing, but not between pre-exposure/conditioning and testing, also has been described (Quintero et al., 2011). Therefore, different contextual changes (both external and internal) applied in different stages of the behavioral procedure may affect the acquisition of latent inhibition (Hall \& Channell, 1986; Quintero et al., 2014; Revillo et al., 2014).

In contrast, no temporal specificity of latent inhibition of CTA was detected when the intake of the taste stimulus was restricted during conditioning. In this condition, both the PE-S and PE-D subgroups showed latent inhibition, and the magnitude of taste aversion over the test days was lower in these subgroups with respect to the nonpreexposed animals, regardless of a change in the temporal context. All these findings can hardly be attributed to a contextual effect on the retrieval of learning (Killcross, 2001; Maren \& Holt, 2000) because the context in the test days (evening session) should not point to the conditioned stimulus-unconditioned stimulus association in the PE-D subgroup without restriction in the intake of the taste stimulus (and therefore this subgroup should express latent inhibition). Rather, the effect of a change of the temporal context on latent inhibition may be due to contextual influences on the association between stimuli (Hall, 1991; Kwok \& Boakes, 2015; Lubow \& De la Casa, 2005; Schmajuk, Lam, \& Gray, 1996).

On the other hand, the behavioral procedure of this study resulted in the delay between the final preexposure trial and conditioning being different for "different" and "same" subgroups. In particular, the PE-D subgroups had a shorter delay compared with the PE-S subgroups, which could influence the results. Nevertheless, because greater temporal proximity between preexposure and conditioning should strengthen the effect of preexposures (De la Casa \& Lubow, 2001), greater latent inhibition should be expected in the "different" subgroups. The results indicate, however, that the subgroup with greater temporal proximity between preexposure and conditioning (PE-D subgroup) showed a disruption of latent inhibition. In addition, this only happened in the subgroup without restriction in the intake of the taste stimulus during conditioning (PE-D and no restriction), although there was also this greater temporal proximity in the restricted subgroup (PE-D and restriction).

In general, we can conclude that the behavioral procedure to induce temporal specificity of latent inhibition of CTA is sensitive to the exposure conditions of the taste stimulus during conditioning. In this experiment, we used 4 days of habituation to the temporal context. Temporal specificity of latent inhibition of CTA under long periods of contextual habituation has been described (Molero-Chamizo, 2013), but a short period seems to be a factor that hinders the contextual effect on taste aversion (Morón et al., 2002). The results of this study complement our knowledge of this phenomenon because they show that an exposure without restriction to the taste stimulus under a long period of contextual habituation facilitates the temporal specificity of the latent inhibition of CTA. However, an external control of the intake behavior during conditioning (a restriction in the intake of the conditioned taste stimulus) under the same period of habituation disrupts this contextual control of the latent inhibition. Thus, long periods of temporal-contextual habituation might facilitate the temporal specificity phenomenon when animals experience a determinate exposure of the conditioned stimulus during conditioning.

Because of the three-stage procedure of the study, the effects of contextual changes on latent inhibition can be attributed to associative mechanisms occurring during conditioning, which may result in changes in the associative strength between 
stimuli (Escobar, Arcediano, \& Miller, 2002; Westbrook, Jones, Bailey, \& Harris, 2000). A restriction in the intake of the conditioned taste stimulus during conditioning might affect the temporal specificity of the latent inhibition of CTA via a modulation of these associative processes. Regarding this modulation, one possibility is that the experience with the taste stimulus is able to modify the way in which the time of day is treated by the memory systems. It is also possible that the arousal level associated with a particular intake can alter the attention directed to contextual cues such as the time of day. Because much remains unknown about the processes of latent inhibition, these results may guide further studies to elucidate the mechanisms involved in the latent inhibition of CTA and the contextual and temporal modulation of this learning.

Acknowledgments This work was supported by the Ministry of Science and Technology (MICYT), Spain, under Grant Number BSO2002-01215, CICYT. The author declares that there is no conflict of interest. The author is grateful to M. Gallo, I. Morón, and M. A. Ballesteros, who contributed to the execution of the experiments. The author wishes to thank the anonymous reviewers for their valuable comments and suggestions, and Francisco Herrero Machaconses for helpful comments on earlier drafts of the manuscript.

\section{References}

Boakes, R. A., Elliot, M., Swinbourne, A. L., \& Westbrook, R. F. (1997). Context dependency of conditioned aversions to water and sweet tastes. Journal of Experimental Psychology: Animal Behavior Processes, 230, 56-57. doi:10.1037/0097-7403.23.1.56

Bonardi, C., Honey, R. C., \& Hall, G. (1990). Context-specificity of conditioning in flavor-aversion learning: Extinction and blocking tests. Animal Learning \& Behaviour, 18, 229-237. doi:10.3758 /BF03205280

Bouton, M. E. (1993). Context, time, and memory retrieval in the interference paradigms of Pavlovian learning. Psychological Bulletin, 114, 80-99. doi:10.1037/0033-2909.114.1.80

Bouton, M. E., Westbrook, R. F., Corcoran, K. A., \& Maren, S. (2006). Contextual and temporal modulations of extinction: Behavioral and biological mechanisms. Biological Psychiatry, 60, 352-360. doi:10.1016/j.biopsych.2005.12

De la Casa, L. G., \& Lubow, R. E. (1995). Latent inhibition in conditioned taste aversion: The roles of stimulus frequency and duration and the amount of fluid ingested during preexposure. Neurobiology of Learning and Memory, 64, 125-132. doi:10.1006 /nlme.1995.1051

De la Casa, L. G., \& Lubow, R. E. (2001). Latent inhibition with a response time measure from a within-subject design: Effects of number of preexposures, masking task, context change, and delay. Neuropsychology, 15, 244-253. doi:10.1037/0894-4105.15.2.244

Escobar, M., Arcediano, F., \& Miller, R. R. (2002). Latent inhibition and contextual associations. Journal of Experimental Psychology: Animal Behavior Processes, 28, 123-136. doi:10.1037/00977403.28.2.123

González, F., Garcia-Burgos, D., \& Hall, G. (2012). Associative and occasion-setting properties of contextual cues in flavor-nutrient learning in rats. Appetite, 59, 898-904. doi:10.1016/j. appet.2012.09.004

González, F., Morillas, E., \& Hall, G. (2015). Latent inhibition in flavorpreference conditioning: Effects of motivational state and the nature of the reinforcer. Learning \& Behavior, 43(4), 376-383. doi:10.3758 /s13420-015-0185-y

Hall, G. (1991). Perceptual and associative learning. Oxford, UK: Clarendon Press.

Hall, G., \& Channell, S. (1986). Context specificity of latent inhibition in taste aversion learning. The Quarterly Journal of Experimental Psychology, 38B, 121-139.

Killcross, S. (2001). Loss of latent inhibition in conditioned taste aversion following exposure to a novel flavour before test. The Quarterly Journal of Experimental Psychology, 54B, 271-288. doi:10.1080 1713932758

Kwok, D. W., \& Boakes, R. A. (2015). Proximal, but not distal, preexposure reduces serial overshadowing in one-trial taste aversion learning. Behavioural Processes, 118, 111-114. doi:10.1016/j. beproc. 2015.06 .006

Lubow, R. E., \& De la Casa, L. G. (2005). There is a time and a place for everything: Bidirectional modulations of latent inhibition by timeinduced context differentiation. Psychonomic Bulletin \& Review, 12(5), 806-821. doi:10.3758/BF03196774

Lukoyanov, N. V., Pereira, P. A., Mesquita, R. M., \& Andrade, J. P. (2002). Restricted feeding facilitates time-place learning in adult rats. Behavioural Brain Research, 134, 283-290. doi:10.1016 /S0166-4328(02)00036-0

Manrique, T., Molero, A., Ballesteros, M. A., Morón, I., Gallo, M., \& Fenton, A. (2004). Time of day-dependent latent inhibition of conditioned taste aversions in rats. Neurobiology of Learning and Memory, 82, 77-80. doi:10.1016/j.nlm.2004.04.003

Manrique, T., Gámiz, F., Morón, I., Ballesteros, M. A., \& Gallo, M. (2009). Peculiar modulation of taste aversion learning by the time of day in developing rats. Developmental Psychobiology, 51, 147157. doi:10.1002/dev.20354

Maren, S., \& Holt, W. (2000). The hippocampus and contextual memory retrieval in Pavlovian conditioning. Behavioural Brain Research, 110, 97-108. doi:10.1016/S0166-4328(99)00188-6

Molero, A., Morón, I., Ballesteros, M. A., Manrique, T., Fenton, A., \& Gallo, M. (2005). Hippocampus, temporal context and taste memories. Chemical Senses, 30, 160-161. doi:10.1093/chemse/bjh163

Molero-Chamizo, A. (2013). Excitotoxic lesion of the hippocampus of Wistar rats disrupts the circadian control of the latent inhibition of taste aversion learning. Brain Research, 1533, 105-113. doi:10.1016/j.brainres.2013.08.030

Morón, I., Manrique, T., Molero, A., Ballesteros, M. A., Gallo, M., \& Fenton, A. (2002). The contextual modulation of conditioned taste aversions by the physical environment and time of day is similar. Learning \& Memory, 9, 18-223. doi:10.1101/lm.52202

Pearce, J. M., \& Bouton, M. E. (2001). Theories of associative learning in animals. Annual Review of Psychology, 52, 111-139. doi:10.1146 /annurev.psych.52.1.111

Quintero, E., Díaz, E., Vargas, J. P., Schmajuk, N., López, J. C., \& De la Casa, L. G. (2011). Effects of context novelty vs. familiarity on latent inhibition with a conditioned taste aversion procedure. Behavioural Processes, 86, 242-249. doi:10.1016/j. beproc.2010.12.011

Quintero, E., Vargas, J. P., Diaz, E., Escarabajal, M. D., Carrasco, M., \& López, J. C. (2014). c-Fos positive nucleus reveals that contextual specificity of latent inhibition is dependent of insular cortex. Brain Research Bulletin, 108, 74-79. doi:10.1016/j. brainresbull.2014.08.008

Revillo, D. A., Gaztañaga, M., Aranda, E., Paglini, M. G., Chotro, M. G., \& Arias, C. (2014). Context-dependent latent inhibition in preweanling rats. Developmental Psychobiology, 56(7), 15071517. doi:10.1002/dev.21236

Rosas, J. M., \& Bouton, M. E. (1997). Renewal of a conditioned taste aversion upon return to the conditioning context after extinction in another one. Learning and Motivation, 28, 216-229. doi:10.1006 /lmot.1996.0960 
Schmajuk, N. A., Lam, Y. W., \& Gray, J. A. (1996). Latent inhibition: A neural network approach. Journal of Experimental Psychology: Animal Behavior Processes, 22, 321-349. doi:10.1037/00977403.22.3.321
Westbrook, R. F., Jones, M. L., Bailey, G. K., \& Harris, J. A. (2000). Contextual control over conditioning responding in a latent inhibition paradigm. Journal of Experimental Psychology: Animal Behavior Processes, 26, 157-173. doi:10.1037/0097-7403.26.2.157 